\title{
Perforating Open Globe Injury
}

National Cancer Institute

\section{Source}

National Cancer Institute. Perforating Open Globe Injury. NCI Thesaurus. Code C118870.

Open globe injury with both entrance and exit wounds in the eyewall (cornea and/or sclera) caused by the same object. 